\title{
CANONICAL CONNECTIONS AND PONTRJAGIN CLASSES
}

\author{
SHOSHICHI KOBAYASHI *
}

In the previous paper [7], we have studied the relationship between the Riemannian connection of an $n$-dimensional Riemannian space $M$ imbedded into the $(n+k)$-dimensional Euclidean space $R^{n+k}$ and the canonical connection in the bundle $P_{n, k}=O(n+k) /\{1\} \times O(k)$ over the Grassmann manifold $M_{n}, k=$ $O(n+k) / O(n) \times O(k)$.

In the first half of the present paper, the relationship between the canonical connections in bundles $P_{n},{ }_{k}, P_{n}^{*},{ }_{k}=O(n+k) / O(n) \times\{1\}, O(n)$ over $M_{n}, k$ and the invariant Riemannian connection on $M_{n},{ }_{k}$ will be discussed. We obtain the holonomy groups of these canonical connections.

In the second half of the paper, we shall study the Pontrjagin classes of manifolds, using exclusively differential forms. To facilitate the calculation, we introduce two types of characteristic cocycles which are closely related to the Pontrjagin cocycles. The duality theorem for the Pontrjagin classes, which has been proved by $\mathrm{Wu}$ Wen-Tsun using the cellular subdivision of the Grassmann manifold $M_{n, k}$ [13], [4], [5], is proved here very easily using the theory of symmetric functions. Our result gives a little bit more precise informations than that of $\mathrm{Wu}$ Wen-Tsun, in the sense that we express the duality theorem as a relation between the Pontrjagin cocycles (in stead of classes) and the normal Pontrjagin cocycles. This may not be interesting for topologists, but may have some value from the differential geometrical point of view. We prove also that the normal Pontrjagin cocycles of a Riemannian space depend only on the Riemannian connection, not on the way how to imbed it. Finally we show that the normal Pontrjagin classes of a manifold $M$ depend only on the differentiable structure of $M$.

The second half of the paper $(\S 4-\S 8)$ can be read independently of the

Received January 9, 1956; revised July 24, 1956.

* Supported by National Science Foundation Grant. 
first half.

\section{$\S 1$. Bundles associated with homogeneous spaces}

Let $G$ be a Lie group acting on a manifold $M$ on the left as a differentiable fransformation group and let $H$ be the isotropic subgroup of $G$ at $x_{0} \in M$. Clearly $H$ is a closed subgroup of $G$, hence a Lie group. Then $M$ is identified with $G / H$ in a natural fashion. Since $H$ acts on $G$ on the right naturally, $G$ is a principal fibre bundle over $G / H=M$ with group $H$ and with the natural projection $p: G \rightarrow G / H$. We shall study the relation between this bundle $G$ over $M$ and the bundle of frames over $M$.

Every element $g$ of $G$ induces a transformation $\delta g$ of $T(M)$, which maps $T_{x_{0}}(M)$ onto $T_{g x_{0}}(M)$ isomorphically. We take $T_{x_{0}}(M)$ as a standard $n$-dimensional vector space $R^{n}(n=\operatorname{dim} M)$. Then $\delta g: R^{n} \rightarrow T_{g x_{0}}(M)$ is a frame at $g x_{0} \in M$, which we shall denote by $u_{g}$. Let $P$ be the bundle of frames over $M$ (with group $G L(n, R)$ ) with projection $\pi$. Then we have the following commutative diagram.

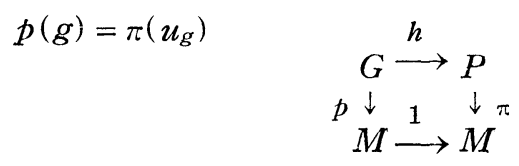

where $h(g)=u_{g}$.

The following proposition is well known.

Proposition 1. $h$ is a one-to-one mapping of $G$ into $P$, if $G$ acts effectively on $M$ and if there exists an affine connection on $M$ which is invariant by $G$.

Under the assumption of Prop. 1, we can consider $G$ as the bundle obtained from $P$ by reducing the structure group $G L(n, R)$ to $H$. And an infinitesimal connection in the bundle $G$ can be considered as an affine connection on $M$ [11].

Consider now a slightly more general case: the case where $G$ is almost effective, i.e., the subgroup $N$ consisting of all elements in $G$ which leave $M$ fixed pointwise is discrete. Note that $N$ is a normal subgroup of $G$ contained in $H$. Now, $G / N$ is a principal fibre bundle over $M$ with group $H / N$, whose projection will be denoted by $p^{\prime}$. The natural map $\psi$ of $G$ onto $G / N$ is a bundle homomorphism with discrete kernel, which mạy be called a semi-isomorphism. The semi-isomorphism $\psi$ induces a one-one correspondence between the set of 
connections in $G$ and the set of connections in $G / N$ [8]. Explicitly, to each connection form $\omega$ on $G / N$, there corresponds the connection form $\psi^{*}(\omega)$ on $G$.

Now suppose that $G$ is almost effective on $M$ and that there exists an affine connection on $M$ invariant by $G$. From Prop. 1 and the above argument, it follows that every connection in the bundle $G$ can be considered as an affine connection on $M$.

We shall apply the above procedure to the case where the orthogonal group $O(n+k)$ in $n+k$ variables acts on the Grassmann manifold $M_{n},{ }_{k}=O(n+k) / O(n)$ $\times O(k)$ in a natural manner. It is easy to see that $O(n+k)$ is almost effective on $M_{n, k}$ (only $I$ and $-I$ act trivially on $M_{n, k}$ ). Since the isotropic subgroup $O(n) \times O(k)$ is compact, there exists an invariant affine connection on $M_{n},{ }_{k}$ [10]. Therefore we can consider every connection in the bundle $O(n+k)$ over $M_{n},{ }_{k}$ as an affine connection on $M_{n}, k$. We shall define the canonical connection in the bundle $O(n+k)$ which corresponds to the invariant Riemannian connection on $M_{n}, k$.

Let $\mathfrak{D}(n+k), \mathfrak{o}(n)$ and $\mathfrak{o}(k)$ be the Lie algebras of $O(n+k), O(n)$ and $O(k)$ respectively. Let $m_{n, k}$ be the orthogonal complement to the subspace $\mathfrak{D}(n)+\mathfrak{D}(k)$ in $\mathfrak{D}(n+k)$ with respect to the Killing-Cartan bilinear form on $\mathfrak{b}(n+k)$. Then

$$
\begin{gathered}
\mathfrak{D}(n+k)=\mathfrak{p}(n)+\mathfrak{o}(k)+\mathfrak{m}_{n, k} \\
a d(s) \cdot \mathfrak{i}_{n}, k \subseteq \mathrm{m}_{n, k} \quad \text { for all } s \in O(n) \times O(k) \\
{\left[\mathfrak{m}_{n}, k, \mathfrak{m}_{n}, k\right] \subseteq \mathfrak{o}(n)+\mathfrak{o}(k) .}
\end{gathered}
$$

Observe that the first two conditions say that $M_{n, k}$ is reductive in the sense of Nomizu [10] and the last one tells that it is moreover symmetric in the sense of $E$. Cartan [2], [10].

Let $\theta$ be the $\mathrm{o}(n+k)$-valued left invariant linear differential form on $O(n+k)$ defined by

$$
\theta(\bar{s})=s^{-1} \bar{s} \quad \bar{s} \in T_{s}(O(n+k)) .
$$

Let $\omega, \eta$ and $\xi$ be the $\mathfrak{o}(n)-, \mathfrak{o}(k)$ - and $\mathrm{m}_{n}, k$-components of the form $\theta$ respectively :

$$
\theta=\omega+\eta+\zeta
$$

It is easy to see that the form $\omega+\eta$ defines a connection in the bundle $O(n+k)$ 
over $M_{n}, k$, which we shall call the canonical connection in the principal fibre bundle $O(n+k)$.

From the fact that $M_{n},{ }_{k}$ is a symmetric space, it follows easily that the canonical connection in $O(n+k)$ corresponds to the invariant affine connection of the 2nd kind [10] on $M_{n}, k$, which is nothing but the invariant Riemannian connection on $M_{n}, k$. In the next section, we shall give an explicit correspondence between the canonical connection in $O(n+k)$ and the invariant Riemannian connection on $M_{n, k}$.

\section{$\S$ 2. Invariant Riemannian connection on $\boldsymbol{M}_{n, k}$}

The Lie algebra $\mathfrak{D}(n+k)$ is the set of skew-symmetric matrices of degree $n+k$ and the spaces $\mathfrak{o}(n), \mathfrak{b}(k)$ and $\mathrm{m}_{n, k}$ are the sets of matrices of the following types respectively:

$$
\left(\begin{array}{ll}
X & 0 \\
0 & 0
\end{array}\right), \quad\left(\begin{array}{cc}
0 & 0 \\
0 & Y
\end{array}\right), \quad\left(\begin{array}{cc}
0 & Z \\
-Z^{t} & 0
\end{array}\right),
$$

where $X$ and $Y$ are skew-symmetric matrices of degree $n$ and $k$ respectively and $Z$ is a matrix of $(n, k)$-type.

Let $x_{0}$ be the point of $M_{n}, k$ which is left fixed by $O(n) \times O(k)$. Then the tangent space $T_{x_{0}}\left(M_{n},{ }_{k}\right)$ can be identified naturally with $\mathrm{m}_{n}, k$, hence a matrix $Z$ can be considered as an element of $T_{x_{0}}\left(M_{n},{ }_{k}\right)$ and conversely. Let $s$ and $s^{\prime}$ be elements of $O(n) \times\{1\}$ and $\{1\} \times O(k)$ respectively. Then the linear transformation of $T_{x_{0}}\left(M_{n}, k\right)$ induced by $s \times s^{\prime} \in O(n) \times O(k)$ corresponds to the following matrix transformation of $\mathrm{m}_{n, k}$ :

$$
\mathrm{m}_{n},{ }_{k} \in z \longrightarrow\left(s \times s^{\prime}\right) \cdot z \cdot\left(s \times s^{\prime}\right)^{-1} \in \mathrm{m}_{n},{ }_{k} .
$$

Let $A$ and $\mathrm{B}$ be the matrices of degree $n$ and $k$ respectively which corresponds to $s$ and $s^{\prime}$. Then

$$
\left(\begin{array}{cc}
A & 0 \\
0 & B
\end{array}\right)\left(\begin{array}{cc}
0 & Z \\
-Z^{t} & 0
\end{array}\right)\left(\begin{array}{cc}
A & 0 \\
0 & B
\end{array}\right)^{-1}=\left(\begin{array}{cc}
0 & A Z B^{t} \\
-\left(A Z B^{t}\right)^{t} & 0
\end{array}\right) .
$$

Therefore the linear transformation $s \times s^{\prime}$ of $T_{x_{0}}\left(M_{n}, k\right)$ is expressed, in terms of matrices, as follows:

$$
Z \longrightarrow \bar{Z} \equiv A Z B^{t}
$$


If

$$
Z=\left(z_{r}^{i}\right), \quad Z=\left(\bar{z}_{r}^{i}\right), \quad A=\left(a_{j}^{i}\right), \quad B=\left(b_{r}^{q}\right),
$$

where $i, j=1, \ldots, n$ and $q, r=1, \ldots, k$, then

$$
\bar{z}_{r}^{i}=\sum a_{j}^{i} b_{b}^{r} z_{q}^{j} .
$$

Consider $A$ and $B$ as linear transformations of the vector spaces $V_{n}$ and $V_{k}$ with bases $e_{1}, \ldots, e_{n}$ and $f_{1}, \ldots, f_{k}$ respectively and identify the tensor product $V_{n} \otimes V_{k}$ with the space of matrices $Z$ as follows :

$$
e_{i} \otimes f_{r} \longleftrightarrow Z_{r}^{i},
$$

where $Z_{r}^{i}$ is the matrix $\left(z_{r}^{i}\right)$ of $(k, n)$-type with

$$
\begin{array}{ll}
z_{q}^{j}=1 \quad \text { if } \quad j=i \text { and } q=r \\
z_{q}^{j}=0 \quad \text { otherwise. }
\end{array}
$$

Then the above transformation $Z \rightarrow A Z B^{t}$ can be identified with the tensor product (the Kronecker product) of $A$ and $B$. We have shown the

Proposition 2. Let $P$ be the bundle of frames over $M_{n, k}$ and $h$ the natural semi-isomorphism of $O(n+k)$ into $P$ (see $\S 1)$. Let $\tau$ be the natural homomorphism of $O(n) \times O(k)$ onto $O(n) \otimes O(k)$; then we have the following commutative diagram:

$$
\begin{aligned}
& O(n+k) \times(O(n) \times(O(k)) \longrightarrow O(n+k) \\
& \downarrow h \times \tau \quad \downarrow h \\
& P \times G L(n k, R) \stackrel{\rho}{\longrightarrow} \quad P
\end{aligned}
$$

where $\varphi$ is the group multiplication in $O(n+k)$ and $\rho$ is the right multiplication by the structure group $G L(n k, R)$.

Let $P^{*}=h(O(n+k))$. Then $P^{*}$ is a principal fibre bundle over $M_{n}, k$ with the structure group $O(n) \otimes O(k)$ and the above proposition can be stated as follows.

Proposition 2'. A pair of mappings $h$ and $\tau$ gives a semi-isomorphism of the bundle $O(n+k)$ onto the bundle $P^{*}$, i.e., the following diagram is commutative:

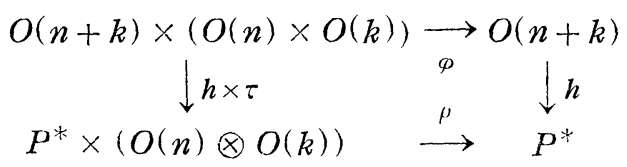


Let $\omega^{*}\left(\right.$ resp. $\left.\eta^{*}\right)$ be the $\mathfrak{o}(n)$-valued (resp. $\mathfrak{o}(k)$-valued) linear differential form on $P^{*}$ corresponding to the form $\omega$ (resp. $\eta$ ) on $O(n+k)$ (see $\S 1$ ). The forms $\omega^{*}$ and $\eta^{*}$ can be considered as skew-symmetric matrix valued differential forms on $P^{*}$. Let $I_{n}$ (resp. $I_{k}$ ) be the unit matrix of degree $n$ (resp. $k$ ). Then $\omega^{*} \otimes I_{k}+I_{n} \otimes \eta^{*}$ is an $\mathfrak{n}(n k)$-valued linear differential form on $P^{*}$. From Prop. $2^{\prime}$, we derive the

Proposition 3. The following diagram is commutative:

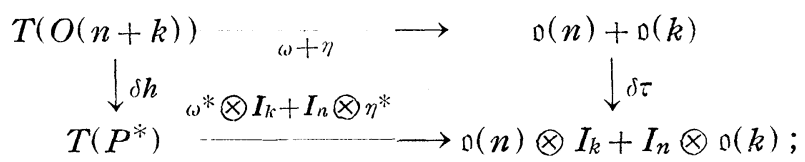

that is, the form $\omega^{*} \otimes I_{k}+I_{n} \otimes \eta^{*}$ defines the connection in $P^{*}$ which corresponds to the canonical connection in the bundle $O(n+k)$.

Remark. The differential $\delta \tau$ of the group homomorphism $\tau$ of $O(n) \times O(k)$ onto $O(n) \otimes O(k)$ induces an algebra isomorphism of $\mathfrak{D}(n)+\mathfrak{D}(k)$ onto $\mathfrak{D}(n) \otimes I_{k}$ $+I_{n} \otimes \mathfrak{o}(k)$.

The above defined connection in $P^{*}$ is evidently an invariant affine connection on $M_{n},{ }_{k}$ whose homogeneous holonomy group is a subgroup of $O(n) \otimes O(k)$. Let $\zeta^{*}$ be the form on $P^{*}$ corresponding to the $\mathfrak{m}_{n}, k$-valued form $\zeta$ on $O(n+k)$. Then the form of soudure on $P$ [7], restricted on $P^{*}$, is $\zeta^{*}$ (under the identification of $R^{n k}$ with $\left.m_{n, k}\right)$. Then the Maurer-Cartan equation on $O(n+k)$ gives

$$
d \zeta_{r}^{i}=-\sum \omega_{j \wedge}^{i} \zeta_{r}^{j}-\sum \eta_{q \wedge}^{r} \zeta_{q}^{i}
$$

If we make use of matrix notations, this can be written as follows :

$$
d \zeta^{*}=-\left(\omega^{*} \otimes I_{k}+I_{n} \otimes \eta^{*}\right) \zeta^{*},
$$

which shows that the connection in $P^{*}$ has no torsion. In general, if an affine connection on a Riemannian manifold has no torsion and its homogeneous holonomy group is contained in the orthogonal group, then it is the Riemannian connection. Hence we have shown explicitly the

Theorem 1. The canonical connection in the principal fibre bundle $O(n+k)$ over $M_{n}, k$ corresponds naturally to the invariant Riemannian connection on $M_{n}, k$. 


\section{$\S 3$. Product of two connections}

Let $P_{1}$ and $P_{2}$ be principal fibre bundles over the same manifold $M$ and with groups $G_{1}$ and $G_{2}$ respectively. The direct product $P_{1} \times P_{2}$ can be considered in a natural manner as a principal fibre bundle over $M \times M$ with group $G_{1} \times G_{2}$. The part of $P_{1} \times P_{2}$ over the diagonal $\Delta(M \times M) \equiv\{(x, x) \in M \times M\}$ is a principal fibre bundle over $M(\cong \Delta(M \times M))$ with group $G_{1} \times G_{2}$, which we shall denote by $P_{1} \circ P_{2}$. Let $\omega_{i}$ be the $g_{i}$-valued linear differential form on $P_{i}$ defining a connection in $P_{i}$ for $i=1,2$, where $g_{i}$ is the Lie algebra of $G_{i}$. Then $\mu_{1}^{*}\left(\omega_{1}\right)+$ $\mu_{2}^{*}\left(\omega_{2}\right)$ defines a connection in $P_{1} \times P_{2}$, where $\mu_{i}$ is the natural projection of $P_{1} \times P_{2}$ onto $P_{i}$. Its restriction on $P_{1} \circ P_{2}$ defines a connection in $P_{1} \circ P_{2}$, which we call the product of the connections $\omega_{1}$ and $\omega_{2}$.

Let $c$ be a curve in $M$ starting from $x_{0}$ and ending at $x_{1}$ and let $u_{i}$ be any point of $P_{i}$ such that $\pi_{i}\left(u_{i}\right)=x_{0}$, where $\pi_{i}$ is the projection of $P_{i}$ onto $M$. Let $c\left(u_{i}\right)$ be the point of $P_{i}$ obtained by parallel displacement of $u_{i}$ along $c$ with respect to the connection defined by $\omega_{i}$. Since $\left(u_{1}, u_{2}\right)$ is a point of $P_{1} \circ P_{2}$ over $x_{0}$, we can define similarly $c\left(u_{1}, u_{2}\right)$, i.e., the point of $P_{1} \circ P_{2}$ obtained by parallel displacement of $\left(u_{1}, u_{2}\right)$ along $c$ with respect to the connection defined by the product of $\omega_{\mathrm{i}}$ and $\omega_{2}$. Then we obtain easily

$$
c\left(u_{1}, u_{2}\right)=\left(c\left(u_{1}\right), c\left(u_{2}\right)\right)
$$

Suppose now that $c$ is a closed curve starting from $x_{0}$. Then there exists a unique element $s_{i} \in G_{i}$ such that

$$
c\left(u_{i}\right)=u_{i} s_{i}
$$

it is by definition an element of the holonomy group with reference point $u_{i}$ associated with the curve $c$. It follows evidently that

$$
c\left(u_{1}, u_{2}\right)=\left(u_{1}, u_{2}\right)\left(s_{1}, s_{2}\right) .
$$

Hence

Proposition 4. Let $h$ be the holonomy group with reference point $u_{i}$ of the connection defined by $\omega_{i}(i=1,2)$ and $h$ the holonomy group with reference point $\left(u_{1}, u_{2}\right)$ of the product of connections $\omega_{1}$ and $\omega_{2}$. Then

(1) $h \subseteq h_{1} \times h_{2}$;

(2) The natural projection $\varphi_{i}: h_{1} \times h_{2} \rightarrow h_{i}$ maps $h$ onto $h_{i}(i=1,2)$.

Remark. $h$ is not necessarily equal to $h_{1} \times h_{2}$. 
Let $P_{n, k}\left(\right.$ resp. $\left.P_{n, k}^{*}\right)$ denote $O(n+k) /\{1\} \times O(k)($ resp. $O(n+k) / O(n) \times\{1\})$ which is a principal fibre bundle over $M_{n},_{k}$ with group $O(n)(\operatorname{resp} . O(k))$. We apply the above argument to the case where $P_{1}=P_{n, k}$ and $P_{2}=P_{n}^{*},{ }_{k}$. We shall show that the principal fibre bundle $O(n+k)$ over $M_{n, k}$ is isomorphic to $P_{n}, k^{\circ} P_{n}^{*}, k$. Let $\nu_{1}$ (resp. $\nu_{2}$ ) be the natural projection of $O(n+k)$ onto $P_{n},{ }_{k}$ (resp. $\left.P_{n}^{*},{ }_{k}\right)$. Then the map $\nu_{1} \times \nu_{2}$ of $O(n+k)$ into $P_{n}, k \times P_{n}^{*},{ }_{k}$ defined by

$$
\left(\nu_{1} \times \nu_{2}\right)(s)=\left(\nu_{1}(s), \nu_{2}(s)\right) \quad s \in O(n+k)
$$

will give an isomorphism of $O(n+k)$ onto $P_{n}, k^{\circ} P_{n}^{*},{ }_{k}$. Let $\pi, \pi_{1}, \pi_{2}$ be the projections of the bundles $O(n+k), P_{n}, k, P_{n, k}^{*}$ onto $M_{n, k}$ respectively. Then

$$
\pi_{1}^{\circ} \nu_{1}(s)=\pi_{2} \circ \nu_{2}(s)=\pi(s) \quad \text { for all } s \in O(n+k),
$$

which shows that $\nu_{1} \times \nu_{2}$ maps $O(n+k)$ into $P_{n, k} \circ P_{n}^{*}, k$.

Suppose that $\left(\nu_{1} \times \nu_{2}\right)(s)=\left(\nu_{1} \times \nu_{2}\right)\left(s^{\prime}\right)$ for some $s, s^{\prime} \in O(n+k)$. From $\nu_{i}(s)=\nu_{i}\left(s^{\prime}\right)(i=1,2)$, it follows that there exist $s_{1} \in O(n) \times\{1\}$ and $s_{2} \in\{1\} \times$ $O(k)$ such that $s^{\prime}=s s_{1}$ and $s^{\prime}=s s_{2}$. Since $O(n) \times\{1\}$ and $\{1\} \times O(k)$ intersect only at the unit, we get that $s_{1}=s_{2}=$ the unit. Hence $s=s^{\prime}$, which proves that $\nu_{1} \times \nu_{2}$ is a one-to-one map. Let $u_{1}$ and $u_{2}$ be arbitrary points of $P_{n, k}$ and $P_{n}^{*},{ }_{k}$ such that $\pi_{1}\left(u_{1}\right)=\pi_{2}\left(u_{2}\right)$. Let $s_{1}$ and $s_{2}$ be elements of $O(n+k)$ such that $\nu_{i}\left(s_{i}\right)$ $=u_{i}$ for $i=1,2$. Then

$$
\pi\left(s_{1}\right)=\pi_{1} \circ \nu_{1}\left(s_{1}\right)=\pi_{2} \circ \nu_{2}\left(s_{2}\right)=\pi\left(s_{2}\right) .
$$

Hence there exist elements $s_{3} \in O(n) \times\{1\}$ and $s_{4} \in\{1\} \times O(k)$ such that $s_{1}=s_{2} s_{3} s_{1}$. Put $s=s_{1} s_{1}^{-1}=s_{2} s_{3}$. Then

$$
\begin{aligned}
& \nu_{1}(s)=\nu_{1}\left(s_{1} s_{4}^{-1}\right)=\nu_{1}\left(s_{1}\right)=u_{1}, \\
& \nu_{2}(s)=\nu_{2}\left(s_{2} s_{3}\right)=\nu_{2}\left(s_{2}\right)=u_{2} .
\end{aligned}
$$

This completes the proof of the following

Proposition 5. The bundle $O(n+k)$ over $M_{n, k}$ is naturally isomorphic to the bundle $P_{n},{ }_{k} \circ P_{n}^{*},{ }_{k}$.

We have shown in the previous paper [7] that the $\mathfrak{v}(n)$-component $\omega$ of the left invariant linear differential form $\theta=\omega+\eta+\zeta$ on $O(n+k)$ induces an $\mathfrak{D}(n)$ valued differential form on $P_{n}, k$, which we denote also by $\omega$ and which defines a connection (called the canonical connection) in the bundle $P_{n, k}$ over $M_{n}, k$. Similarly we can prove that the $\mathfrak{D}(k)$-component $\eta$ of $\theta$ induces an $\mathfrak{D}(k)$-valued 
differential form on $P_{n}^{*}, k$, which we shall denote by the same letter $\eta$ and which defines a connection in $P_{n}^{*},{ }_{k}$ (by definition, the canonical connection in $P_{n}^{*},{ }_{k}$ ). Then

Proposition 6. Under the natural isomorphism between the bundles $O(n+k)$ over $M_{n, k}$ and $P_{n}, k_{k} \circ P_{n}^{*}, k$, the canonical connection in $O(n+k)$ corresponds to the product of the canonical connections in $P_{n}, k$ and $P_{n, k}^{*}$.

Now we shall study the holonomy group of the canonical connections in $O(n+k), P_{n}, k$ and $P_{n}^{*}, k$. Because of Theorem 1 , the study of the canonical connection in $O(n+k)$ can be reduced to that of the invariant Riemannian connection on $M_{n}, k$. Since $M_{n}, k$ is an irreducible symmetric space in the sense of E. Cartan, the restricted holonomy group of the invariant Riemannian connection on $M_{n},{ }_{k}$ coincides with the connected component of the unit of the linear isotropic subgroup of the group of isometries [2], [10], in this case, $S O(n) \otimes$ $S O(k)$.

In order to obtain the (non-restricted) holonomy group, we have to investigate the canonical connection more carefully. By the above argument and Theorem 1 , the restricted holonomy group of the canonical connection in $O(n+k)$ is $S O(n) \times S O(k)$. Taking the unit of $O(n+k)$ as a reference point, we consider the set $P_{0}$ of all points in $O(n+k)$ which can be joined to the unit by horizontal curves [1], [8] with respect to the canonical connection in $O(n+k)$. The set $P_{0}$ is a principal fibre bundle over $M_{n, k}$ whose structure group is the holonomy group of the canonical connection [1], [8]. Since $P_{0}$ is arcwise connected, it is a submanifold of $S O(n+k)$. On the other hand, it is of the same dimension as $S O(n+k)$, because the connected component of the structure group of $P_{0}$ has the same dimension as $S O(n+k) \cap(O(n) \times O(k))$. As easily seen, $P_{0}$ cannot be a proper open submanifold of $S O(n+k)$. Hence $P_{0}$ coincides with $S O(n+k)$. The holonomy group of the canonical connection in $O(n+k)$ is, therefore, $S O(n+k) \cap(O(n) \times O(k))$. From Prop. 4 we obtain immediately the holonomy group of the canonical connection in $P_{n, k}$ (resp. $P_{n}^{*},{ }_{k}$ ). Thus we have obtained the

THEOREM 2. The holonomy groups of the canonical connections in $O(n+k)$, $P_{n, k}$ and $P_{n}^{*},{ }_{k}$ are respectively $S O(n+k) \cap(O(n) \times O(k)), O(n)$ and $O(k)$.

From the above theorem it follows that the holonomy group of the invariant 
Riemannian connection on $M_{n}, k$ is the image of $S O(n+k) \cap(O(n) \times O(k))$ under the natural isomorphism of $O(n) \times O(k)$ onto $O(n) \otimes O(k)$ and is decomposed into two connected components: one contains the unit and the other contains the following element:

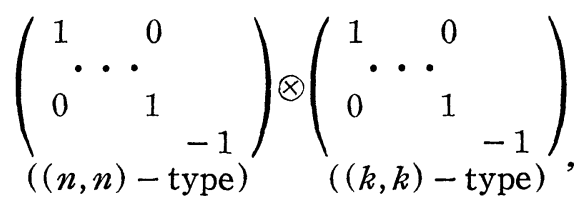

whose determinant is obviously $(-1)^{n+k}$. As it is well known, $M_{n, k}$ is orientable if and only if $n+k$ is even.

\section{$\S 4$. Curvature forms of canonical connections}

From the Maurer-Cartan equation of $\theta=\omega+\eta+\zeta$, we obtain

$$
\begin{aligned}
& d \omega=-\frac{1}{2}[\omega, \omega]-\frac{1}{2}[\zeta, \zeta]_{1} \\
& d \eta=-\frac{1}{2}[\eta, \eta]-\frac{1}{2}[\zeta, \zeta]_{2},
\end{aligned}
$$

where $[\zeta, \zeta]_{1}$ and $[\zeta, \zeta]_{2}$ are respectively the $\mathfrak{D}(n)$ - and $\mathfrak{D}(k)$-components of $[\zeta, \zeta]$. Therefore the curvature forms $\Omega_{1}$ and $\Omega_{2}$ of the canonical connections in $P_{n, k}$ and $P_{n, k}^{*}$ are given by ${ }^{1)}$

$$
\begin{aligned}
& \Omega_{1}=-\frac{1}{2}[\zeta, \zeta]_{1} \\
& \Omega_{2}=-\frac{1}{2}[\zeta, \zeta]_{2} .
\end{aligned}
$$

Now we shall calculate the curvature form of the invariant Riemannian connection on $M_{n, k}$ defined by

$$
\gamma=\omega^{*} \otimes I_{k}+I_{n} \otimes \eta^{*} .
$$

We observe that, for any matrices $A, B$ of degree $n$ and any matrix $C$ of degree $k$,

$$
\left[A \otimes I_{k}, B \otimes I_{k}\right]=[A, B] \otimes I_{k}
$$

Hence

$$
\left[A \otimes I_{k}, I_{n} \otimes C\right]=A \otimes C-A \otimes C=0 .
$$

$$
[\gamma, \gamma]=\left[\omega^{*}, \omega^{*}\right] \otimes I_{k}+I_{n} \otimes\left[\eta^{*}, \eta^{*}\right]
$$

1) More precisely, $\Omega_{1}$ (resp. $\Omega_{2}$ ) is the form on $P_{n, k}$ (resp. $P_{n}^{*},{ }_{k}$ ) induced from the form $-\frac{1}{2}[\zeta, \zeta]_{1}$ (resp. $\left.-\frac{1}{2}[\zeta, \zeta]_{2}\right)$ on $O(n+k)$. 
and

$$
\begin{aligned}
d \gamma+\frac{1}{2}[\gamma, \gamma] & =d \omega^{*} \otimes I_{k}+I_{n} \otimes d \eta^{*}+\frac{1}{2}\left[\omega^{*}, \omega^{*}\right] \otimes I_{k}+\frac{1}{2} I_{n} \otimes\left[\eta^{*}, \eta^{*}\right] \\
& =\Omega_{1}^{*} \otimes I_{k}+I_{n} \otimes \Omega_{2}^{*},
\end{aligned}
$$

where $\Omega_{1}^{*}$ is the form on $p^{*}$ corresponding to $\Omega_{i}$.

\section{$\S 5$. Characteristic classes}

Let $P$ be an arbitrary principal fibre bundle over a manifold $M$ with Lie group $G$ and with projection $\pi$. Let $g$ be the Lie algebra of G. A polynomial function $f$ defined on $g$ is invariant by $G$ if

$$
f(a d(s) \cdot g)=f(g) \quad \text { for all } g \in g \text { and } s \in G .
$$

Suppose there is given a connection in $P$ and let $\Omega$ be its curvature form. Then $\Omega$ is a $g$-valued 2 -form on $P$. The composite $f(\Omega)$ is a real valued differential form of degree $2 r$ if $f$ is a homogeneous polynomial of degree $r$. From the property

$$
\begin{array}{r}
\Omega\left(\bar{u}_{1} \bar{s}_{1}, \bar{u}_{2} \bar{s}_{2}\right)=s^{-1} \Omega\left(\bar{u}_{1}, \bar{u}_{2}\right) s \quad \text { for all } \bar{u}_{1}, \bar{u}_{2} \in T_{u}(P), \\
\bar{s}_{1}, \bar{s}_{2} \in T_{s}(G),
\end{array}
$$

we conclude that, if $f$ is invariant by $G$, then there exists a unique differential form $f(\Omega)^{*}$ on $M$ such that

$$
\pi^{*}\left(f(\Omega)^{*}\right)=f(\Omega) .
$$

It is known [3], [4] that $f(\Omega)^{*}$ is a closed form and the cohomology class to which it belongs is independent of the choice of connections. The cohomology class of $f(\Omega)^{*}$ is a characteristic class of the bundle $P$.

Remark 1. The form $f(\Omega)$ is always the coboundary of a certain form on $P$; however $f(\Omega)^{*}$ is not, in general, cohomologous to zero in $M$.

Remark 2. If $M$ is a Riemannian (resp. Hermitian) manifold and $P$ is the bundle of orthogonal frames (resp. unitary frams) over $M$, then the Pontrjagin classes (resp. Chern classes) are characteristic classes of $P$ in the above defined sense.

\section{§ 6. Symmetric functions}

First we recall some known results in the theory of symmetric functions. We shall consider three types of symmetric functions of $n$ quantities $a_{1}, \ldots, a_{n}$. 
The symmetric functions $p_{1}, \ldots, p_{n}$ of the first type are associated with the equation whose roots are the reciprocals of the quantities $a_{i}$;

$$
f(x) \equiv \Pi\left(1-a_{i} x\right)=1-p_{1} x+p_{2} x^{2}-\ldots+(-1)^{n} p_{n} x^{n} .
$$

The function $p_{r}$ is the sum of the $\left(\begin{array}{l}n \\ r\end{array}\right)$ products of $r$ different quantities $a_{i}$. Define $p_{r}=0$ for $r>n$.

The symmetric functions $q_{1}, \ldots, q_{n}, \ldots$ of the second type can be obtained by formal expansion of $1 / f(x)$;

$$
\begin{aligned}
1 / f(x) & =1 / \Pi\left(1-a_{r} x\right)=\Pi\left(1+a_{r} x+a_{r}^{2} x^{2}+\ldots\right) \\
& =1+q_{1} x+q_{2} x^{2}+\ldots+q_{n} x^{n}+\ldots .
\end{aligned}
$$

Then $q_{r}$ is the sum of the homogeneous products of degree $r$ of the quantities $a_{1}, \ldots, a_{n}$.

The symmetric functions $t_{1}, \ldots, t_{n}, \ldots$ of the third type are defined by

$$
t_{r}=\sum_{i=1}^{n} a_{i}^{r} .
$$

The following formulae are fundamental [9].

$$
\begin{aligned}
& p_{r}-p_{r-1} q_{1}+p_{r-2} q_{2}-\ldots+(-1)^{r} q_{r}=0
\end{aligned}
$$

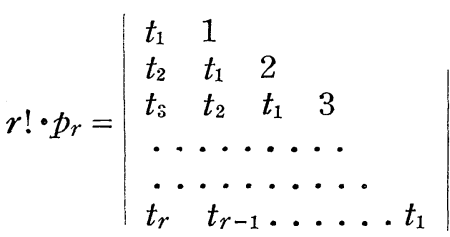

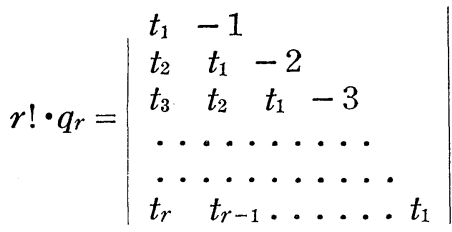

Let $B=\left(b_{j}^{i}\right)$ be a matrix of degree $n$ over the field of real numbers and let $C$ be a non-singular matrix of degree $n$ over the field of complex numbers such that $\left(a_{j}^{i}\right) \equiv A=C^{-1} B C$ is a triangular matrix :

Put

$$
a_{j}^{i}=0 \quad \text { if } \quad i>j .
$$

$$
a_{1}=a_{1}^{1}, \ldots, a_{n}=a_{n}^{n}
$$

We shall express the symmetric functions $p_{r}, t_{r}$ of the $n$ quantities $a_{1}, \ldots$, 
$a_{n}$ in terms of elements $b_{j}^{i}$ of the matrix $B$. Put

$$
p_{r}^{\prime}(B)=\frac{1}{r !} \sum_{i, j \delta}\left(i_{1}, \ldots, i_{r} ; j_{1}, \ldots, j_{r}\right) b_{j_{1}}^{i_{1}} \ldots b_{j_{r}}^{i_{r}}
$$

where $\delta\left(i_{1}, \ldots, i_{r} ; j_{1}, \ldots, j_{r}\right)$ is the generalized Kronecker $\delta$. From $A=$ $C^{-1} B C$, it follows that $p_{r}^{\prime}(A)=p_{r}^{\prime}(B)$. This fact can be proved as follows. A linear transformation $B$ of the $n$-dimensional vector space $V$ (over the field of complex numbers) induces a linear transformation $B_{r}$ of $\wedge^{r} V$, where $\wedge^{r} V$ is the space of homogeneous elements of degree $r$ of the exterior algebra $\wedge V$ over $V$. Then $p_{r}^{\prime}(B)$ is nothing but the trace of $B_{r}$. This proves our assertion. Now we shall prove that $p_{r}^{\prime}(A)=p_{r}$. Since $A$ is triangular, we have

$$
p_{r}^{\prime}(A)=\frac{1}{r !} \sum_{i_{1} \leq j_{1}, \ldots, i_{r} \leq j_{r} \delta} \delta\left(i_{1}, \ldots, i_{r} ; j_{1}, \ldots, j_{r}\right) a_{j_{1}}^{i_{1}} \ldots a_{j_{r}}^{i_{r}} .
$$

It is easy to see that, if $i_{1} \leqslant j_{1}, \ldots, i_{r} \leqslant j_{r}$, then

$$
\delta\left(i_{1}, \ldots, i_{r} ; j_{1}, \ldots, j_{r}\right)=0 \text { unless } i_{1}=j_{1}, \ldots, i_{r}=j_{r} .
$$

Hence we have that $p_{r}^{\prime}(A)=p_{r}$. Finally we have obtained

$$
p_{r}=\frac{1}{r !} \sum \delta\left(i_{1}, \ldots, i_{r} ; j_{1}, \ldots, j_{r}\right) b_{j_{1}}^{i_{1}} \ldots b_{j_{r}}^{i_{r}} .
$$

Next we shall calculate the symmetric functions $t_{r}$ of the quantitites $a_{1}, \ldots$, $a_{n}$. Since $A^{r} \equiv A A \ldots A=C^{-1} B^{r} C$, we have

$$
\operatorname{trace}\left(A^{r}\right)=\operatorname{trace}\left(B^{r}\right) \text {. }
$$

It is clear that $t_{r}=\operatorname{trace}\left(A^{r}\right)$. On the other hand,

$$
\operatorname{trace}\left(B^{r}\right) \sum b_{i_{2}}^{i_{1}} b_{i_{3}}^{i_{2}} \ldots b_{i_{1}}^{i_{r}}
$$

Hence

$$
t_{r}=\sum b_{i_{2}}^{i_{1}} b_{i_{3}}^{i_{2}} \ldots b_{i_{1}}^{i_{r}}
$$

Notice that, if $B$ is skew-symmetric and $r$ is odd, then both $p_{r}$ and $t_{r}$ vanish. The definition of $q_{r}^{\prime}(B)$ is clear from (III) and (V).

\section{$\S 7$. Characteristic classes of $\boldsymbol{P}_{n, k}$ and $\boldsymbol{P}_{n, k}^{*}$}

Let $X$ be an arbitrary element of the Lie algebra $\mathfrak{D}(n)$. We define $p_{r}^{\prime}(X)$ as in the preceding section. Then $p_{r}^{\prime}$ is a polynomial invariant by $O(n)$. Replacing $X$ by the curvature form $\Omega_{1}$ of the canonical connection in $P_{n}, k$, we obtain

$$
p_{r}^{\prime}\left(\Omega_{1}\right)=\frac{1}{r !} \sum \delta\left(i_{1}, \ldots, i_{r} ; j_{1}, \ldots, j_{r}\right) \Omega_{1}^{i_{1}} j_{1} \wedge \ldots \wedge \Omega_{1}^{i_{r}} j_{r} .
$$


Let $\widetilde{p}_{r}$ be the form on $M_{n},{ }_{k}$ such that

$$
\pi_{1}^{*}\left(\widetilde{p}_{r}\right)=p_{r}^{\prime}\left(\Omega_{1}\right) .
$$

Similarly we obtain

$$
t_{r}^{\prime}\left(\Omega_{1}\right)=\sum \Omega_{1 i_{2} \wedge}^{i_{1}} \Omega_{1 i_{3}}^{i_{2}} \wedge \cdots \wedge \Omega_{1 i_{1}}^{i_{r_{1}}}
$$

and define the form $\tilde{t}_{r}$ on $M_{n}, k$ such that

$$
\pi_{1}^{*}\left(\widetilde{t}_{r}\right)=t_{r}^{\prime}\left(\Omega_{1}\right) .
$$

We define also the form $\widetilde{q}_{r}$ on $M_{n, k}$ such that

$$
\pi_{1}^{*}\left(\widetilde{q}_{r}\right)=q_{r}^{\prime}\left(\Omega_{1}\right) .
$$

The differential forms $\widetilde{p}_{r}, \widetilde{t}_{r}$ and $\widetilde{q}_{r}$ on $M_{n, k}$ are all closed and their cohomology classes are independent of choice of connections in $P_{n, k}$.

Let $Y$ be an arbitrary element of the Lie algebra $\mathfrak{D}(k)$. We define $p_{r}^{\prime *}(Y)$ in the same way (just replacing $n$ by $k$ ). Replacing $Y$ by the curvature form $\Omega_{2}$ of the canonical connection in $P_{n, k}^{*}$, we obtain

$$
P_{r}^{\prime *}\left(\Omega_{2}\right)=\frac{1}{r !} \sum \delta\left(i_{1}, \ldots, i_{r} ; j_{1}, \ldots, j_{r}\right) \Omega_{2 j 1}^{i_{1}} \wedge \ldots \wedge \Omega_{2 j_{r}}^{i r} .
$$

Let $\widetilde{p}_{r}^{*}$ be the form on $M_{n}, k$ such that

$$
\pi_{2}^{*}\left(\tilde{p}_{r}^{*}\right)=p_{r}^{\prime *}\left(\Omega_{2}\right) .
$$

We define similarly the forms $\widetilde{t}_{r}^{*}$ and $\widetilde{q}_{r}^{*}$ on $M_{n}, k$.

Firstly we shall prove that

$$
\tilde{t}_{r}+\widetilde{t}_{r}^{*}=0
$$

For this purpose, it suffices to show that

$$
\pi^{*}\left(\widetilde{t}_{r}\right)+\pi^{*}\left(\widetilde{t}_{r}^{*}\right)=0
$$

where $\pi$ is the natural projection of $O(n+k)$ onto $M_{n}, k$. The left invariant $O(n+k)$-valued differential form $\theta$ on $O(n+k)$ is a skew-symmetric matrix differential form $\left(\theta_{j}^{i}\right)_{i, j=1}, \ldots, n+k$. We have

$$
\begin{array}{ll}
d \theta_{\beta}^{\alpha}=-\sum_{\gamma=1}^{n} \theta_{\gamma \wedge}^{\alpha} \theta_{\beta}^{\Upsilon}-\sum_{\lambda=n+1}^{n+k} \theta_{\lambda \wedge}^{\alpha} \theta_{\beta}^{\lambda}, & \alpha, \beta=1, \ldots, n \\
d \theta_{\mu}^{\lambda}=-\sum_{\nu=n+1}^{n+k} \theta_{\nu \wedge}^{\lambda} \theta_{\mu}^{\nu}-\sum_{\alpha=1}^{n} \theta_{\alpha \wedge}^{\lambda} \theta_{\mu}^{\alpha}, & \lambda, \mu=n+1, \ldots, n+k .
\end{array}
$$

Hence

$$
\begin{aligned}
& \pi^{*}\left(\tilde{t}_{r}\right)=(-1)^{r} \sum\left(\theta_{\lambda_{1} \wedge}^{\alpha_{1}} \theta_{\alpha_{2}}^{\lambda_{1}}\right) \wedge\left(\theta_{\lambda_{2} \wedge}^{\alpha_{2}} \theta_{\alpha_{3}}^{\lambda_{2}}\right) \wedge \cdots \wedge\left(\theta_{\lambda_{r} \wedge}^{\alpha_{r}} \theta_{\alpha_{1}}^{\lambda_{r}}\right) \\
& \pi^{*}\left(\widetilde{t}_{r}^{*}\right)=(-1)^{r} \sum\left(\theta_{\alpha_{2} \wedge}^{\lambda_{1}} \theta_{\lambda_{2}}^{\alpha_{2}}\right) \wedge\left(\theta_{\alpha_{3} \wedge}^{\lambda_{2} \wedge} \theta_{\lambda_{3}}^{\alpha_{3}}\right) \wedge \cdots \wedge\left(\theta_{\alpha_{1} \wedge}^{\lambda_{r}} \wedge \theta_{\lambda_{1}}^{\alpha_{1}}\right),
\end{aligned}
$$


where the summations are taken over the indices $\alpha_{1}, \ldots, \alpha_{r}$ which run from 1 to $n$ and the indices $\lambda_{1}, \ldots, \lambda_{r}$ which run from $n+1$ to $n+k$. It is now evident that $\pi^{*}\left(\widetilde{t}_{r}\right)=-\pi^{*}\left(\tilde{t}_{r}^{*}\right)$. We have proved the following duality theorem.

THEOREM 3. $\tilde{t}_{r}+\tilde{t}_{r}^{*}=0$.

Now we shall find the corresponding relationship between $\widetilde{p}_{r}$ and $\widetilde{p}_{r}^{*}$. From the formula (III) in $\S 6$ and Theorem 3 , it follows that

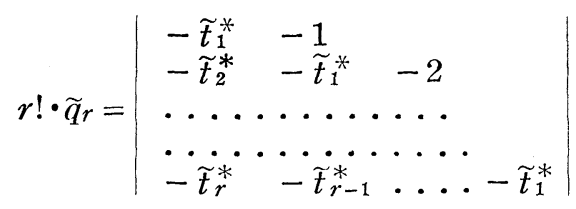

Hence

$$
\widetilde{q}_{r}=(-1)^{r} \widetilde{p}_{r}^{*}
$$

Now, from (I) in $\S 6$, we obtain the

ThEOREM $3^{\prime} . \quad \widetilde{p}_{r}+\widetilde{p}_{r-1} \widetilde{p}_{1}^{*}+\widetilde{p}_{r-2} \widetilde{p}_{2}^{*}+\ldots+\widetilde{p}_{r}^{*}=0$.

Remark. As we have seen in the preceding section, the forms $\tilde{p}_{r}, \tilde{p}_{r}^{*}, \tilde{t}_{r}$ and $\tilde{t}_{r}^{*}$ vanish identically if $r$ is odd.

\section{$\S 8$. Characteristic classes of a manifold}

Let $M$ be an $n$-dimensional Riemannian manifold imbedded isometrically in the $(n+k)$-dimensional Euclidean space. Let $P$ be the bundle of (tangent) orthogonal frames over $M$ and let $P^{*}$ be the bundle of normal orthogonal frames over $M$. Then $P$ and $P^{*}$ are principal fibre bundles over $M$ with group $O(n)$ and $O(k)$ respectively. Let $h_{1}$ be the natural bundle map of $P$ into $P_{n}, k$. We have shown in the previous paper [7] that the connection in $P$ induced by $h_{1}$ from the canonical connection in $P_{n, k}$ is nothing but the Riemannian connection on $M$. Let $h_{2}$ be the natural bundle map of $P^{*}$ into $P_{n, k}^{*}$. Then $h_{2}$ and the canonical connection in $P_{n}^{*},{ }_{k}$ induce a connection in $P^{*}$, which we shall call the normal connection. It seems that the normal connection has been considered implicitly in classical differential geometry.

Both $h_{1}$ and $h_{2}$ induce the same mapping $h$ of the base space $M$ into the base space $M_{n}, k$. Put

$$
p_{r}(M)=h^{*}\left(\widetilde{p}_{r}\right), \quad q_{r}(M)=h^{*}\left(\widetilde{q}_{r}\right), \quad t_{r}(M)=h^{*}\left(\widetilde{t}_{r}\right),
$$




$$
p_{r}^{*}(M)=h^{*}\left(\widetilde{p}_{r}^{*}\right), \quad q_{r}^{*}(M)=h^{*}\left(\widetilde{q}_{r}^{*}\right), \quad t_{r}^{*}(M)=h^{*}\left(\widetilde{t}_{r}^{*}\right) .
$$

The differential forms $p_{r}(M)$ and $p_{r}^{*}(M)$ on $M$ are called the $2 r$-th Pontrjagin cocycle ${ }^{2)}$ and the $2 \boldsymbol{r}$-th normal Pontrjagin cocycle of the imbedded Riemannian manifold $M$. The cohomology classes of $p_{r}(M)$ and $p_{r}^{*}(M)$ are called the $2 r$-th Pontrjagin class and the $2 r$-th normal Pontrjagin class of $M$ respectively. From Theorems 3 and 3 , we obtain the

Theorem 4. $t_{r}(M)+t_{r}^{*}(M)=0$,

$$
p_{r}(M)+p_{r-1}(M) \cdot p_{1}^{*}(M)+\ldots+p_{r}^{*}(M)=0 .
$$

Since the forms $t_{r}(M)$ are completely determined by the curvature form of the Riemannian connection on $M$, so are the forms $t_{r}^{*}(M)$. Since the forms $p_{r}^{*}(M)$ and $q_{r}^{*}(M)$ are polynomials of $t^{*}(M)$ (see (II) and (III) in $\S 6$ ), they depend only on the curvature form of the Riemannian connection on $M$. Hence

THeOREm 5. The differential forms $p_{r}^{*}(M), q_{r}^{*}(M)$ and $t_{r}^{*}(M)$ are all invariants of the Riemannian connection on $M$.

We shall explain the meaning of the above theorem. Suppose $j: M \rightarrow R^{n+k}$ and $j^{\prime}: M \rightarrow R^{n+k^{\prime}}$ and $j^{\prime}: M \rightarrow R^{n+k^{\prime}}$ be isometrical imbeddings of an $n$-dimensional Riemannian space $M$. If $k^{\prime} \leq k$, then we may consider $j^{\prime}$ as an isometrical imbedding of $M$ into $R^{n+k}$. In general, there may not exist any motion $\psi$ of $R^{n+k}$ such that $j^{\prime}=\psi \cdot j$. The above theorem states that the forms $p_{r}^{*}(M), q_{r}^{*}(M)$, $t_{r}^{*}(M)$ do not depend on $j$ but depend only on the Riemannian connection on $M$.

As we have remarked in $\S 5$, the cohomology classes of $p_{r}(M), q_{r}(M), t_{r}(M)$ are differentiable invariants of $P$, hence they are differentiable invariants of $M$. From Theorem 4, it follows that the cohomology class of $t_{r}^{*}(M)$ is also a differentiable invariant of $M$. Since the forms $p_{r}^{*}(M), q_{r}^{*}(M)$ are polynomials of $t_{r}^{*}(M)$ (see (II) and (III) in $\S 6$ ), their cohomology classes are also differentiable invariants of $M$.

Theorem 6. The cohomology classes of $p_{r}(M), q_{r}(M), t_{r}(M), p_{r}^{*}(M), q_{r}^{*}(M)$ and $t_{r}^{*}(M)$ depend only on the differentiable structure of the manifold $M$.

Remark. We understand by "differentiable structure" " $C^{\infty}$-differentiable

2) Our definition of Pontrjagin cocycles (and normal Pontrjagin cocycles) is different from that of $[4,5]$ by constant factors. In the following theorem 4 , these constant factors are cancelled out. 
structure". However the above theorem is true for the $C^{3}$-differentiable structure.

\section{BIBLIOGRAPHY}

[1] Ambrose, W. and Singer, I. M., A theorem on holonomy, Trans. Amer. Math. Soc., 75 (1955), 428-443.

[2] Cartan, E., Sur une classe remarquable d'espaces de Riemann, Bull. Soc. Math. France, 54 (1926), 214-268; 55 (1927), 114-134.

[ 3 ] Cartan, H., Notion d'algèbre différentiable etc., Colloque de topologie, Bruxelles (1950), 15-27, 57-71.

[4] Chern, S-S., Lecture notes in Princeton(1951).

[5] - L La géométrie des sous-variétés d'un espace euclidien à plusieurs dimensions, Enseignement math. (1955), 26-46.

[6] Ehresmann, C., Les connexions infinitésimales dans un espace fibré différentiable, Colloque de topologie, Bruxelles (1950), 29-55.

[ 7 ] Kobayashi, S., Induced connections and imbedded Riemannian spaces, Nagoya Math. J., 10 (1956), 15-25.

[8] - Theory of connections (thesis), to appear.

[9] Littlewood, D. E., Theory of group characters, Oxford (1940).

[10] Nomizu, K., Invariant affine connections on homogeneous spaces, Amer. J. Math., 76 (1954), 33-65.

[11] — Reduction theorem for connections and its application etc., Nagoya Math., J. 9 (1955), 57-66.

[12] Pontrjagin, L., Some topological invariants of closed Riemannian manifolds, Izvestiya Akad. Nauk SSSK, ser. Math., 13 (1949), 125-162; Amer. Math. Soc. Trans., No. 49.

[13] Wu Wen-Tsun, Sur les espaces fibrés, Paris Hermann (1952).

\section{University of Washington}

